\title{
BMJ Open Study protocol for a multisite randomised controlled trial of a rehabilitation intervention to reduce participation restrictions among female breast cancer survivors
}

Courtney J Stevens (D) , ${ }^{1,2}$ Mark T Hegel, ${ }^{2}$ Marie Anne Bakitas, ${ }^{3}$ Martha Bruce, ${ }^{1,2}$ Andres Azuero, ${ }^{3}$ Maria Pisu, ${ }^{4}$ Mary Chamberlin, ${ }^{2,5}$ Kimberly Keene, ${ }^{6}$ Gabrielle Rocque, ${ }^{7}$ Daphne Ellis, ${ }^{1}$ Tiffany Gilbert, ${ }^{1}$ Jamme L Morency, ${ }^{8}$ Robin M Newman, ${ }^{9}$ Megan E Codini, ${ }^{8}$ Karen E Thorp, ${ }^{8}$ Sarah M dos Anjos, ${ }^{10}$ Danielle Z Cloyd, ${ }^{3}$ Jennifer Echols, ${ }^{3}$ Ashley N Milford, ${ }^{3}$ Stacey A Ingram, ${ }^{11}$ Jasmine Davis, ${ }^{11}$ Kathleen Doyle Lyons (D) ${ }^{1,2}$

To cite: Stevens CJ, Hegel MT, Bakitas MA, et al. Study protocol for a multisite randomised controlled trial of a rehabilitation intervention to reduce participation restrictions among female breast cancer survivors. BMJ Open 2020;10:e036864. doi:10.1136/ bmjopen-2020-036864

- Prepublication history and additional material for this paper are available online. To view these files, please visit the journal online (http://dx.doi. org/10.1136/bmjopen-2020036864).

Received 07 January 2020 Revised 14 January 2020 Accepted 16 January 2020

D) Check for updates

(c) Author(s) (or their employer(s)) 2020. Re-use permitted under CC BY-NC. No commercial re-use. See rights and permissions. Published by BMJ.

For numbered affiliations see end of article.

\section{Correspondence to} Dr Kathleen Doyle Lyons; kathleen.d.lyons@dartmouth. edu

\section{ABSTRACT}

Introduction Many breast cancer survivors report an inability to fully participate in activities of daily living after completing cancer treatment. Reduced activity participation is linked to negative consequences for individuals (eg, depression, reduced quality of life) and society (reduced workforce participation). There is currently a lack of evidence-based interventions that directly foster cancer survivors' optimal participation in life roles and activities. Pilot study data suggest rehabilitation interventions based on behavioural activation (BA) and problem-solving treatment (PST) can facilitate posttreatment role resumption among breast cancer survivors. Methods and analysis This protocol describes a multisite randomised controlled trial comparing a 4-month long, ninesession BA and PST-informed rehabilitation intervention (BA/ PS) against a time-matched, attention control condition. The overall objective is to assess the efficacy of BA/PS for enhancing breast cancer survivors' activity participation and quality of life over time. A total of 300 breast cancer survivors reporting participation restrictions after completing curative treatment for stage 1-3 breast cancer within the past year will be recruited across two sites (DartmouthHitchcock Medical Center and University of Alabama at Birmingham). Assessments are collected on enrolment (T1) and 8 (T2), 20 (T3) and 44(T4) weeks later.

Ethics and dissemination Study procedures are approved by the Committee for the Protection of Human Subjects at Dartmouth College, acting as the single Institutional Review Board of record for both study sites (STUDY 00031380). Results of the study will be presented at national meetings and submitted for publication in peerreviewed journals.

Trial registration number NCT03915548; Pre-results.

\section{INTRODUCTION}

While the 5-year relative survival rate for breast cancer has risen to $89 \%,{ }^{1}$ many breast
Strengths and limitations of this study

- Rigorous design including randomisation, attention control condition and adequate power allows testing the efficacy of the intervention.

- Fidelity to treatment will be highly monitored.

- One of the first studies to directly target activity and role resumption (ie, overcoming participation restrictions) of breast cancer survivors.

- Telephone delivery increases accessibility of the intervention, ideally allowing recruitment of participants who are unable (for physical or practical reasons) to return to the cancer centre for rehabilitation.

- Participants and interventionists are not blinded to the treatment condition/group assignment.

cancer survivors report an inability to fully participate in vocational, social, physical and instrumental activities of daily living after completing cancer treatment. ${ }^{2}$ The activities most often affected by cancer and its treatment are those requiring sustained time and effort outside the home (eg, working, shopping and social events). ${ }^{3}$

Reduced activity participation is linked to negative consequences at both an individual and societal level. Breast cancer survivors who report lower participation in productive and social activities experience greater depression, ${ }^{4}$ lower quality of life ${ }^{5}$ and shortened survival. ${ }^{67}$ Additionally, the excess employment disability and reduced work productivity experienced by breast cancer survivors ${ }^{8}$ can result in fewer financial and social opportunities for the individuals and lower productivity for society. ${ }^{910}$ Two recent reviews concluded 


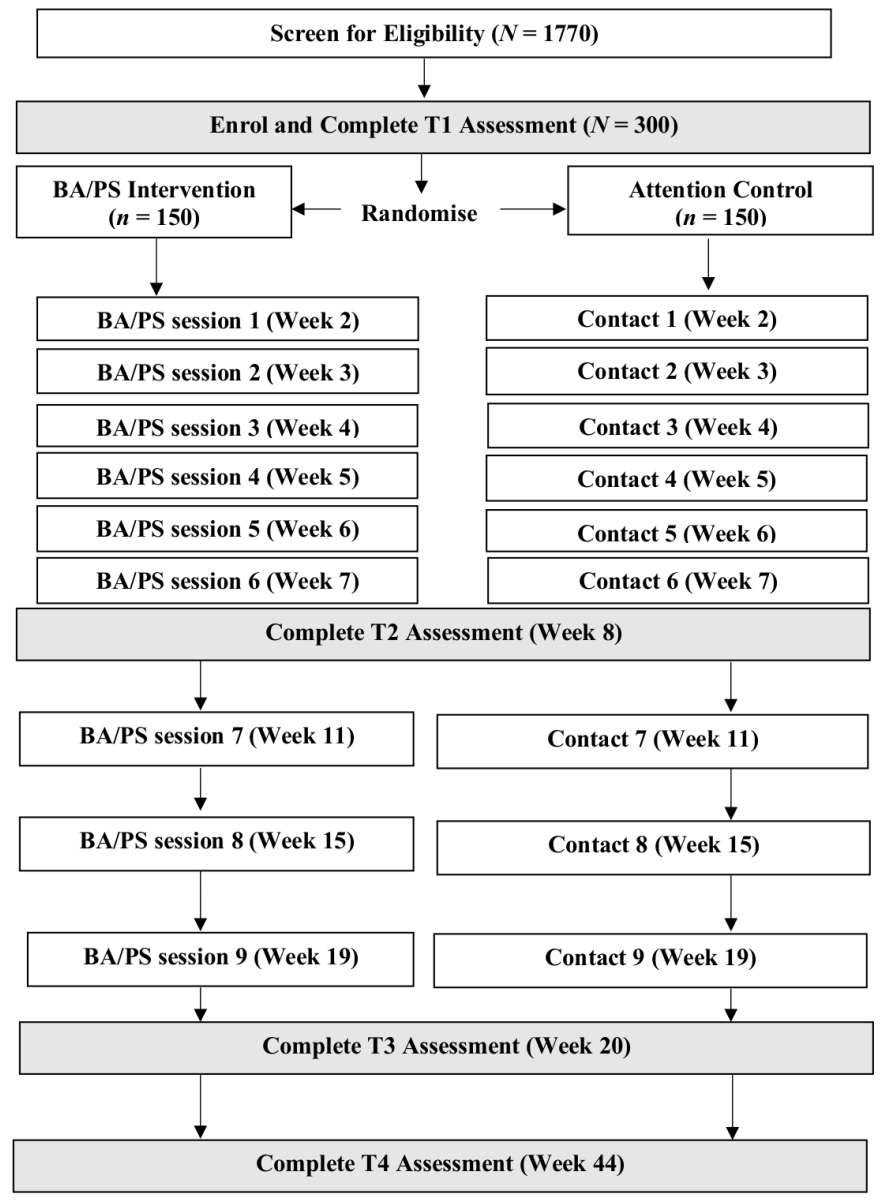

Figure 1 Study design. BA/PS, behavioural activation/ problem-solving.

that there is a lack of evidence-based interventions that directly foster cancer survivors' optimal participation in life roles and activities. ${ }^{1112}$

Our team developed a theory-driven intervention designed to directly foster cancer survivors' role resumption and activity participation. We refer to the intervention as behavioural activation/problem-solving (BA/ PS), as it integrates the cognitive-behavioural therapies of behavioural activation ${ }^{13}$ and problem-solving treatment ${ }^{1516}$ and incorporates concepts from an occupational therapy theory called the person-environmentoccupational performance model ${ }^{17} \mathrm{BA} / \mathrm{PS}$ teaches breast cancer survivors to strategically adapt an activity and the environment in order to make the activity easier, more enjoyable and more sustainable. Over nine sessions, survivors set, adjust and achieve strategic goals that incrementally enhance and optimise their participation in daily activities. The present study builds on three pilot studies that previously demonstrated the feasibility, acceptability and potential efficacy of the brief, practical, telephonedelivered $\mathrm{BA} / \mathrm{PS}$ intervention to fostering functional recovery after breast cancer treatment. ${ }^{18-21}$

\section{Study aims and hypotheses}

The present study (Institutional Review Board (IRB) protocol version V3 dated 10 February 2019) is designed to answer the question, 'Is BA/PS efficacious in enhancing activity participation and quality of life of breast cancer survivors within oneyear of completing curative treatment for Stage 1-3 breast cancer?'

\section{Aim 1}

To test the effect of BA/PS on breast cancer survivors' participation in roles and activities. Compared with attention control participants, BA/PS participants are expected to report greater postintervention participation, activity performance and productivity.

Aim 2

To test the effect of BA/PS on breast cancer survivors' quality of life. Compared with attention control participants, BA/PS participants are expected to report higher postintervention quality of life.

\section{Aim 3 (exploratory)}

To test the effect of BA/PS on coping, goal adjustment and distress outcomes. Compared with attention control participants, BA/PS participants are expected to report greater postintervention adaptive coping, greater goal adjustment and less distress.

\section{METHODS AND ANALYSIS Study design}

This protocol describes a parallel-group, multisite, superiority randomised controlled trial (RCT) with two study arms: (1) the BA/PS intervention condition, which consists of six weekly telephone calls followed by three monthly telephone calls over 4 months, and (2) the attention control condition, which consists of the same amount of study team contact over the same 4-month period, but focuses on providing education via telephone about cancer survivorship topics. See figure 1 for a schematic diagram of all study contacts and assessments.

\section{Study sites}

Participants will be enrolled from the National Cancer Institute-designated comprehensive cancer centres at Dartmouth-Hitchcock Medical Center in New Hampshire and the University of Alabama at Birmingham.

\section{Recruitment and screening}

We will recruit 300 women over the age of 18 reporting participation restrictions (ie, challenges in engaging in productive and/or pleasurable activities that fulfil valued life roles) after completing curative treatment for stage 1-3 breast cancer within the past year. We obtained a Health Insurance Portability and Accountability Act (HIPAA) waiver to allow research staff to screen clinic schedules to identify potential study participants. Research coordinators consult with clinicians to confirm patient eligibility based on clinical characteristics and then clinicians deliver the study brochure to patients. Research coordinators are available in the clinic or by telephone to 
further explain the study to patients, formally screen for eligibility and initiate informed consent procedures.

In order to maximise recruitment, additional recruitment strategies of 'direct-to-consumer' approaches (eg, speaking at support groups, tables at breast cancer awareness rallies), sending letters to potentially eligible participants identified via medical record extraction and/ or working with patient navigators will be employed as needed.

\section{Participants}

Inclusion criteria

Female patients, aged 18 years or older, diagnosed with stage 1-3 breast cancer, within 1year of completing locoregional treatment and/or chemotherapy with curative intent and absence of disease recurrence, are eligible for this study if they are experiencing participation restrictions (ie, a score of $\geq 10$ on the Work and Social Adjustment $\mathrm{Scale}^{22}$ ). There is no upper age limit imposed, as evidence from our pilot studies suggests the intervention is flexible enough to be used with various challenges that occur across the age range. ${ }^{1820}$ Because our sites see a mean of 3.8 males each year with breast cancer, we will enrol only females as there are not enough males to draw sound conclusions generalisable to a male population. This study targets survivors within 1 year of completing curative therapy because we are interested in supporting the middle phase of survivorship (ie, the transition from active treatment towards extended survival). ${ }^{23}$

\section{Exclusion criteria}

Patients are not eligible for this study if they are nonEnglish speaking, experience non-correctable hearing loss, have moderate-severe cognitive impairment (indicated by a score $<3$ on a 6 -item cognitive screener ${ }^{24}$ ), or have a history of severe mental illness (ie, schizophrenia, bipolar disorder), current major depressive disorder, active suicidal ideation or active substance misuse documented within the medical record. Potential participants will not be excluded solely because of medical comorbidities (eg, arthritis, back pain) as this work ultimately aims to develop a generalisable intervention that has broad applicability for cancer survivors experiencing disability (ie, strong external validity); thus, it is necessary to develop and test interventions that can address any type of participation restriction, regardless of its source. Participants will also not be excluded if they are receiving other supportive care or rehabilitation services.

\section{Randomisation}

Participants will be randomly assigned to conditions (1:1) using a computer-generated programme. The randomisation scheme will be stratified by site (Dartmouth and Alabama), time since treatment completion (ie, $<6$ and $>6$ months) and receipt of chemotherapy (yes vs no) and will be blocked within strata (block lengths of 2 and 4 varied randomly). As the central coordinating site, Dartmouth will manage the randomisation process. The
Dartmouth Principal Investigator will be alerted to the assignment when the baseline assessment is completed by the coordinators and will trigger the local interventionist to communicate assignment to the participant and initiate treatment activities.

Data collectors (ie, Dartmouth coordinators) and analysts will be blinded to assignment. Participants will be instructed not to discuss their assignment with the Dartmouth coordinators during the interviews. The data collectors and analysts will remain blind to assignment until the analyses are completed. Participants may choose to withdraw from the study or from the intervention; however, the intervention and control conditions will not be modified for any reason. Participants who wish to withdraw from the intervention will be invited and encouraged to continue to participate in the study and provide outcome assessments.

\section{Intervention}

Our approach to improving activity participation reflects self-regulation models that emphasise alignment between goals and circumstances. ${ }^{25-28}$ Recognition of a discrepancy between one's goals and circumstances leads to either adaptive or maladaptive coping. Adaptive coping can be viewed as efforts to change the activity, environment or self. These efforts manifest themselves in active coping (ie, taking action instead of waiting for problems to disappear), planning (strategically deciding what actions to take) and positive reframing (adjusting expectations and interpretations of events). ${ }^{20}$ Goal adjustment is another self-regulation strategy with two components. ${ }^{29}$ The first component, goal disengagement, prevents the negative emotional consequences of pursuing a futile goal. The second component, goal re-engagement, directs renewed energy towards attainable goals. BA/PS is designed to promote adaptive coping and goal adjustment through a process of strategic goal setting, problem-solving and action planning centred on increasing the ease and enjoyment of activity participation and life roles, leading to lower distress, improved productivity and higher quality of life.

BA/PS teaches survivors to (A) systematically examine the reasons an activity is challenging, (B) set achievable short-term goals that have the potential to improve participation, (C) brainstorm solutions including activity adaptations and environmental modifications, (D) construct and implement a detailed action plan, and (E) evaluate the results and level of goal attainment. The structured process gives participants repeated practice in goal re-engagement that leads them progressively closer to their long-term functional goals.

The intervention was developed over a series of pilot studies. ${ }^{18-21}$ In the first session, the interventionist presents the rationale for $\mathrm{BA} / \mathrm{PS}$, promotes a positive problem orientation and educates the participant about the framework for problem-solving and action planning. The interventionist then administers the Canadian Occupational Performance Measure $(\mathrm{COPM})^{30}$ to elicit 


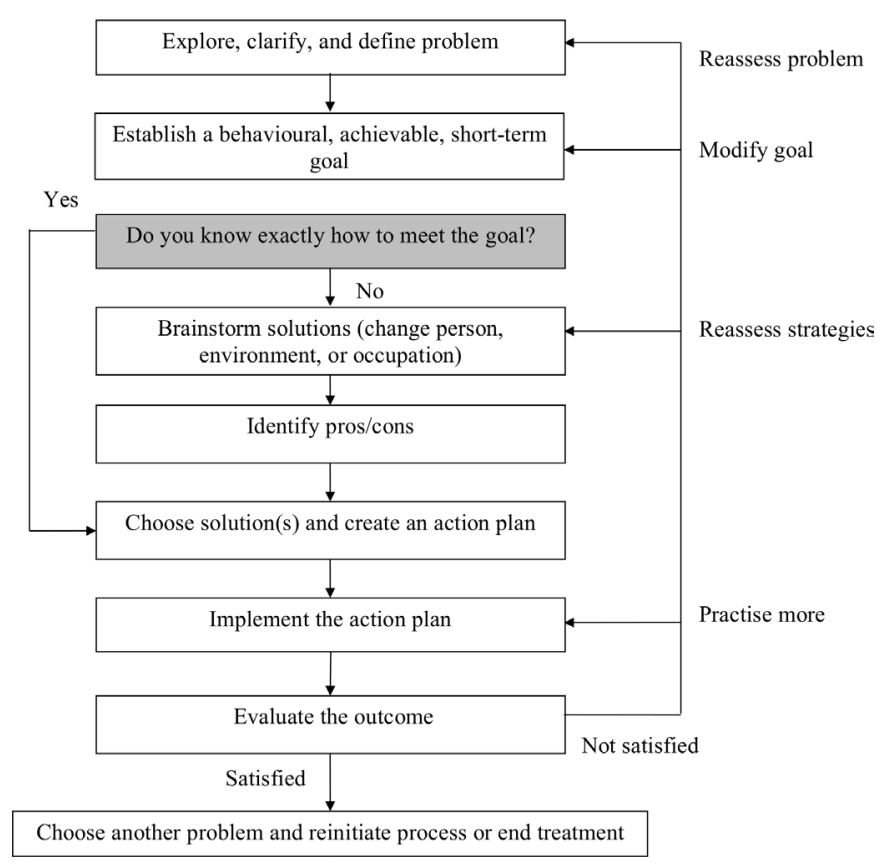

Figure 2 Behavioural activation/problem-solving framework.

participant priorities, motivation and long-term goals. In subsequent sessions, the interventionist guides the participant in using the BA/PS framework (see figure 2) to set a goal, brainstorm solutions to challenges and create a detailed action plan for the coming week. Results of the action plan are used to refine goals and action plans and promote steady progress towards personally selected recovery goals.

\section{Attention control condition}

Using randomisation and a usual care control condition would eliminate threats to internal validity that arise with time and history (ie, participants might improve naturally as time goes by or because of an event occurring at the sites). However, BA/PS participants would be receiving more attention and support than the usual care participants. We recognise that the attention experienced within a weekly telephone call from a warm and supportive therapist could lift the spirits of participants and it is theoretically plausible that feeling more hopeful or supported could allow and encourage participants to become more active and engaged in life. It is important to control for this possibility so that we can determine if it is our specific $\mathrm{BA} / \mathrm{PS}$ intervention, and not general support or attention, that drives any effects seen in our data. Thus, the attention control condition will allow us to account for the effect of time and history as well as other non-specific effects of attention.

Increased attrition can occur if participants feel that an attention placebo is not a meaningful use of time. ${ }^{31}$ As such, we decided to provide education regarding participant-selected cancer survivorship topics (ie, healthy diets, physical activity, lymphoedema management, smoking cessation, fear of recurrence, cognitive challenges, communication with providers and family, fatigue, sleep, hot flashes and night sweats, and work accommodations) during the control telephone contacts. The control condition will match the intervention in terms of the number of sessions, the delivery by telephone and delivery by an occupational therapist. This will allow us to determine the effect of the specific BA/PS elements (ie, strategic goal setting, problem-solving, activity adaptation, environmental modification and action planning) on participation and quality of life.

\section{Assessments}

As the central coordinating site for the study, all data collection will occur from and be managed by Dartmouth staff. Data will be directly entered into the Research Electronic Data Capture (REDCap) software system. ${ }^{32}$ Assessments will be administered via telephone by a research coordinator blinded to group assignment (see figure 1). Participants will complete outcome assessments on enrolment (T1) and at 8 weeks (T2), 20 weeks (T3) and 44 weeks (T4) later (see table 1). The T2 assessment captures the short-term outcomes of the most intensive part of the intervention (ie, after six weekly sessions). The T3 assessment will capture the short-term outcomes at the end of the full intervention. The T4 assessment explores the sustained effect of BA/PS (6 months after BA/PS ends). Participants will be paid US $\$ 25$ for completion of each of the first three assessments and will be paid US $\$ 30$ on completion of the last assessment (total=US $\$ 105$ per participant).

\section{Measures}

\section{Demographics and clinical characteristics}

Enrolled participants are asked to report their age, race, ethnicity, employment status, education level, marital status, insurance status, number of dependent children living at home and household income. Relevant data regarding disease stage, treatment and comorbidities are obtained from the medical record. ${ }^{33}$

\section{Participation satisfaction}

Participation is a construct with two dimensions: satisfaction and ability. Disability scholars have argued that the subjective assessment of satisfaction with daily routines and activities is a defining feature of participation. ${ }^{34}$ As such, the Patient-Reported Outcomes Measurement Information System (PROMIS) Satisfaction with Social Roles and Activities Short Form $8 a^{3536}$ is used to measure our primary outcome. This 8-item self-report scale, which has been previously validated for use with cancer survivors, ${ }^{37}$ assesses satisfaction with abilities regarding routine, work, leisure, family and social activities. Items are rated using a 5-point Likert scale (1='not at all' to $5=$ 'very much'). Scores on this measure are calculated as the sum of all items, thus possible scores range from 8 to 40 , with higher values representing greater satisfaction with ability to participate in social roles and activities. 
Table 1 Measures and data collection schedule

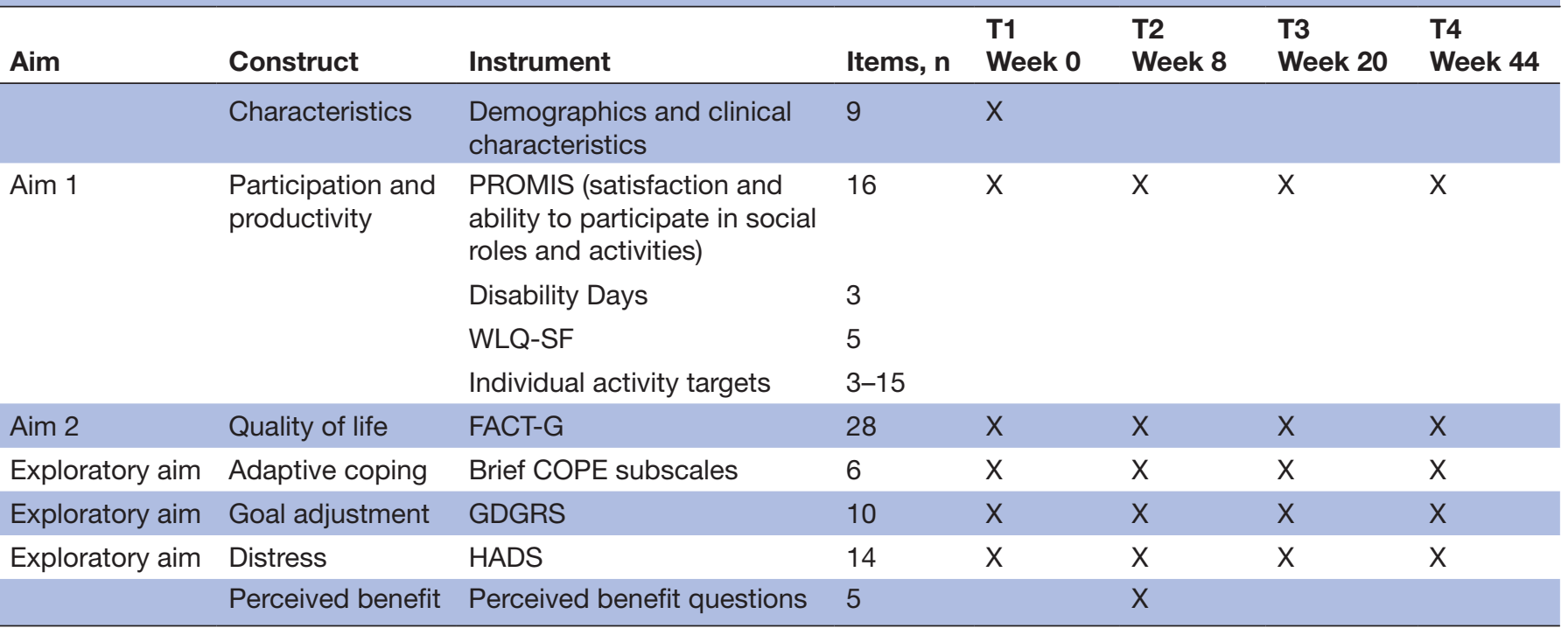

Research assistants will administer the outcome assessment battery by telephone on enrolment (T1), after completion of the most intensive portion of the intervention (T2), after completion of the full intervention (T3) and 6 months after completion of the intervention (T4).

Brief COPE, Brief Coping Orientation to Problems Experienced; FACT-G, Functional Assessment of Cancer Therapy-General; GDGRS, Goal Disengagement and Goal Reengagement Scale; HADS, Hospital Anxiety and Depression Scale; PROMIS, Patient-Reported Outcomes Measurement Information System; WLQ-SF, Work Limitations Questionnaire-Short Form.

\section{Participation ability}

The PROMIS Ability to Participate in Social Roles and Activities Short Form $8 a^{3536}$ scale is used to measure the ability aspect of participation. This 8-item self-report scale, developed in conjunction with the satisfaction scale, as described above, assesses the ability to participate in activities as desired at work, during leisure and with friends and family. Items are rated using a 5-point Likert scale ( $1=$ 'always' to $5=$ 'never'). Scores on this measure are calculated as the sum of all items, thus possible scores range from 8 to 40, with lower values representing greater difficulty participating in social roles and activities. Our team added an item to this scale in order to solicit participant perceptions of the degree to which their challenges are related to cancer or its treatment.

\section{Productivity}

We use relevant questions from the Disability Days section of the Medical Expenditure Panel Survey $(M E P S)^{838}$ to capture days missed from work and lost household productivity in the past 30 days. For all participants, including those who are not employed or are on leave, lost household productivity is assessed by counting the days spent in bed, defined in MEPS as half days or more spent in bed because of physical illness or injury, or mental or emotional problems. Days missed from work (or school) are defined in MEPS as half days or more missed from work (or school). Women who are neither students nor employed outside the home are only asked to report on lost household productivity.

The Work Limitations Questionnaire-Short Form (WLQ$S F)^{39}$ is also used to assess productivity. The subscale scores represent the percentage of time in the previous
2 weeks that participants may be limited in performance in the dimensions of time, physical, mental or interpersonal, and output. Items are rated using a 5-point Likert scale $(100 \%=$ 'difficult all of the time', $50 \%=$ 'difficult some of the time', $0 \%=$ 'difficult none of the time'). An overall WLQ Productivity Loss Score is the weighted sum of the four subscale scores and indicates the percentage decrement in productivity (ie, scores range from $0 \%$ to $100 \%$ ). The WLQ has been used in cancer survivor populations ${ }^{40-42}$ and has demonstrated high validity and reliability. ${ }^{39} 43$

\section{Individual activity targets}

The $C O P M^{30}$ is used with each participant to elicit up to five individual activity recovery goals during the first and last study sessions. Participants rate each targeted activity using Likert scales ranging from 1 to 10 for three characteristics of performance $(1=$ 'not able to do it' to $10=$ 'able to do it extremely well'), importance ( $1=$ 'not important at all' to $10=$ 'extremely important') and satisfaction with the activity ( $1=$ 'not satisfied at all' to $10=$ 'extremely satisfied') . Higher scores indicate greater participation and occupational performance.

\section{Quality of life}

The Functional Assessment of Cancer Therapy-General (FACT$G)$ is a 27-item self-report measure of health-related quality of life specifically designed for patients with breast cancer. ${ }^{445}$ The items of the tool assess perceived well-being in physical, social, emotional and functional domains. Items are rated using a 5-point Likert scale $(0=$ 'not at all' to $4=$ "very much'). Total scores on this measure are calculated as the sum of all items, thus 
possible scores range from 0 to 108 , with higher values representing greater functioning in each domain. Subscale scores are derived in each domain as well; scores for the physical well-being, social/family well-being and functional well-being subscales range from 0 to 28 and scores on the emotional well-being subscale range from 0 to $24 .{ }^{45}$

\section{Coping}

The Brief COPE ${ }^{46}$ is a self-report scale used to assess use of coping strategies for difficult situations. The Brief COPE has been shown to have excellent psychometric properties among patients with cancer including evidence of construct, convergent and concurrent criterion validity. ${ }^{47}$ Our pilot research has suggested that BA/PS increases participants' use of active coping, planning and positive reframing. ${ }^{20}$ These coping styles are measured by three subscales of the Brief COPE. To minimise respondent burden, we will not administer the 28-item Brief COPE, but will instead use these three subscales of active coping, planning and positive reframing, each of which has two items. Items are rated using a 4-point Likert scale $(0=$ 'I haven't been doing this at all', $3=$ 'I've been doing this a lot'). Scores on each subscale are calculated as the sum of the two items, thus possible scores range from 0 to 6 , with higher values representing greater use of active coping strategies.

\section{Goal adjustment}

The Goal Disengagement and Goal Reengagement Scale $(G D G R S)^{48}$ is a 10 -item self-report scale that measures two aspects of goal adjustment: goal disengagement (ie, the general inclination to relinquish untenable goals) and goal re-engagement (ie, commit to new goals). Items are rated using a 5-point Likert scale (1='almost never true', $5=$ 'almost always true'). Items $1-4$ summed to create the goal disengagement subscale (items 2 and 3 are reverse scored), thus scores range from 4 to 20 , with higher scores representing greater ability to disengage from a goal. Likewise, items 5-10 are summed to create the goal re-engagement subscale, thus scores range from 6 to 30 , with higher scores representing greater ability to re-engage with new goals.

\section{Distress}

The Hospital Anxiety and Depression Scale (HADS) is a 14-item self-report measure of depressive and anxiety symptoms specifically designed for use with medical patients. ${ }^{37} 49$ Items are rated on a 4-point Likert scale ranging from 1 to 4 with different anchors for each item (eg, 1='definitely', 4='not at all'). Even numbered items are summed to create the depression subscale and odd numbered items are summed to create the anxiety subscale; higher scores on each subscale indicate greater depression and anxiety symptoms, respectively. Items $1,3,5,8,10,11$ and 13 are reverse scored. Scores on each subscale range from 7 to 28 .

\section{Perceived benefit}

Our team developed six questions modelled after items used in a recent rehabilitation $\mathrm{RCT}^{50}$ to help us describe the credibility and utility of the BA/PS intervention and control conditions. Using a 3-point scale $(0=$ 'not at all', $1=$ 'some', $2=$ 'a great deal'), participants are asked to rate the degree to which the programme helped them gain confidence, reduce distress, adjust habits and routines, set goals and exercise. These items should also function as a manipulation check; at minimum, participants assigned to the BA/PS intervention should report higher levels of benefit on the goal-setting item as the control condition does not include goal setting as an active component.

\section{Patient and public involvement}

Cancer survivors were indirectly involved in the design of the study by virtue of their participation in prior descriptive studies in which we solicited their perspectives on participation restrictions. Further, prior pilot studies solicited feedback from cancer survivors regarding the proposed intervention and study procedures (eg, feasibility of the assessments, timing of recruitment). The feedback in these preliminary studies informed the research question and design of the current study. When the current study nears completion, we will ask completing participants if they would like to join an advisory board that would provide input in the interpretation and dissemination of results.

\section{Statistical analysis, sample size and power estimates}

Using a time-averaged difference approach, ${ }^{51}$ at a corrected significance level of $0.05 / 3=0.017$, and with three time points after baseline, the required sample size to test for the minimally important effects of BA/PS on participation (PROMIS scales) ranges from 63 to 117 in each arm to achieve $80 \%$ power for within-subject correlations ranging from 0.2 to 0.8 . The required sample size to test for the minimally important effect of BA/PS on quality of life (overall FACT-G score) ranges from 45 to 83 in each arm in order to achieve $80 \%$ power for withinsubject correlations ranging from 0.2 to 0.8 .

We will examine the balance between study groups with respect to baseline characteristics using descriptive statistics and measures of effect size. We will examine the patterns of missing data due to dropout, and whether baseline characteristics are associated with dropout. Baseline factors showing non-trivial imbalances between groups or that are predictive of dropout will then be used as adjusting covariates in the longitudinal group comparisons.

Our objective is to compare functional recovery across conditions over time in terms of participation (PROMIS scales; Aim 1), productivity (Disability Days and WLQSF; Aim 1), quality of life (FACT-G; Aim 2) and coping style, distress and goal adjustment (Brief COPE, HADS and GDGRS; Aim 3). A longitudinal model fitted with linear mixed methods will be used for each outcome (see figure 3). The focus of inference will be the between-group 


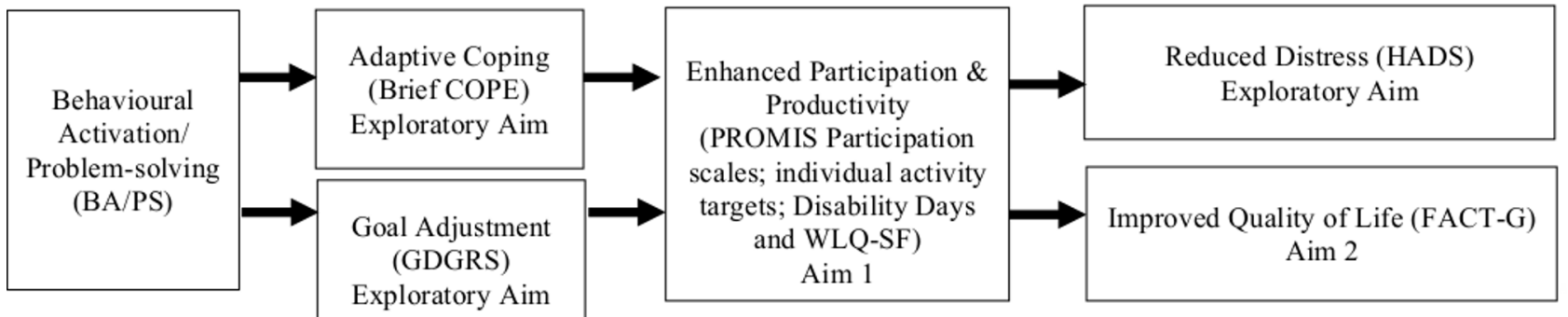

Exploratory Aim

Figure 3 Conceptual model of outcomes. Brief COPE, Brief Coping Orientation to Problems Experienced; COPM, Canadian Occupational Performance Measure; FACT-G, Functional Assessment of Cancer Therapy-General; GDGRS, Goal Disengagement and Goal Reengagement Scale; HADS, Hospital Anxiety and Depression Scale; PROMIS, Patient-Reported Outcomes Measurement Information System; WLQ-SF, Work Limitations Questionnaire-Short Form.

difference in outcome trajectories over the study time points, modelled by a time by group interaction. The overall treatment effect will be computed as the betweengroup difference in change from baseline (change from $\mathrm{T} 1$ averaged over T2-T4) estimated with a linear contrast.

Mixed modelling techniques and covariate adjustment will reduce the impact of missing data, as the missingness is not assumed completely at random (MCAR) but conditionally (on the covariates) at random (ie, missing at random (MAR), a milder assumption). ${ }^{52}$ Should dropout exceed the $20 \%$ allowed by the sample size, non-parametric multiple imputation ${ }^{53}$ will be employed to determine the robustness of the conclusions for the main analyses under the milder MAR assumption. Due to the non-invasive, supportive nature of the BA/PS intervention, as well as the attention control condition, we do not expect to encounter non-random dropout. However, we will examine the tracking system records and logs with regard to dropout to determine the main reasons for dropout. If sufficient indication of non-random dropout is found, sensitivity analyses under different assumptions for the missing data mechanism will be conducted, following the methodology described by Molenberghs and Kenward ${ }^{54}$ in which the missing data mechanism needs to be modelled explicitly.

A false discovery rate (FDR) approach ${ }^{55}$ will be used to adjust inferential results for multiple inferences on the same body of data, separately for the primary analyses and the exploratory analyses. The FDR is the expected proportion of true null differences among those that are declared 'significant'. The FDR will be set at $10 \%$. All research products will disclose the number of inferences conducted and whether outcomes were primary or exploratory.

The BA/PS intervention is designed to be flexible and responsive to the needs of a diverse group of breast cancer survivors and our diverse sample will provide an opportunity to identify any unanticipated moderator effects. Therefore, we will explore whether there are subgroups of participants who benefited most and least during the study, as per the primary outcomes. We will use recursive partitioning, that is, classification and regression trees (CART),${ }^{56}$ to conduct moderator analyses. CART is a nonparametric modelling approach that allows extracting of multivariate profiles from a sufficiently large data set under minimal modelling assumptions, based on values of an outcome and participant characteristics. Because CART is data driven, these analyses are considered exploratory.

To avoid assuming that missing outcome data due to dropout are missing completely at random (ie, MCAR), a random forest-based algorithm ${ }^{53}$ will be used to generate three imputed data sets comprising baseline characteristics, group assignment and longitudinal outcome variables. CART modelling will be implemented on each imputed data set and results will be compared as a form of sensitivity analysis. The target variables for the CART modelling will be the average change from baseline in each primary outcome. Regression tree models for these target variables will be fitted using as predictors the group assignment and selected baseline characteristics. These baseline characteristics will include indicators of pertinent subpopulations of interest: site, race, income, age, and so on. We will implement the conditional inference approach ${ }^{57}$ to fit the tree models and use repeated 10-fold cross-validation to determine the final tree size. The tree model is a decision-tree-like structure that is interpreted based on the characteristics of the resulting groups of participants.

Primary analyses will use an intent-to-treat approach. All available data from all participants who undergo randomisation will be included in the analyses according to the group assigned, regardless of any postrandomisation protocol deviations.

\section{ETHICS AND DISSEMINATION \\ Informed consent}

All subjects are provided a consent form describing the study with sufficient information to make an informed decision about participation in the study. Formal consent is obtained and documented by study research coordinators who orally review the form and address questions and concerns before participants undergo any study procedures. The consent process continues throughout the study as both coordinators and interventionists review study procedures and affirm continued desire to continue during each study contact. 


\section{Confidentiality}

Participant data are kept confidential and managed according to the requirements of the HIPAA of 1996. A study ID number is used in place of identifiable participant information on all study documents. Study data are collected and managed using REDCap ${ }^{32}$ electronic data capture tools hosted at Dartmouth-Hitchcock by the Clinical and Translational Science Institute (SYNERGY). REDCap is a secure, web-based software platform designed to support data capture for research studies, providing (1) an intuitive interface for validated data capture; (2) audit trails for tracking data manipulation and export procedures; (3) automated export procedures for seamless data downloads to common statistical packages; and (4) procedures for data integration and interoperability with external sources.

\section{Data and safety monitoring plan}

The informed consent process, the recruitment process and the timeliness and quality of the data are continuously monitored by the principal investigator, the IRB and the Data Safety Monitoring and Accrual Committee (DSMAC) of the Norris Cotton Cancer Center. The DSMAC is a multidisciplinary committee charged with overseeing the monitoring of participant safety, the conduct and progress of research protocols and the validity and integrity of the data of clinical trials at Norris Cotton Cancer Center and its subsites. The Committee meets quarterly to review accrual rates and information for studies that have accrued participants and includes representatives from medical oncology, haematology, radiation oncology, investigational pharmacy, biostatistics and clinical research administration. All adverse events will be tracked by the study team and reported to the IRB and the DSMAC. All protocol modifications will be reviewed by the IRB and communicated to relevant stakeholders as determined by the IRB. The DSMAC has the authority to suspend or to recommend termination all research activities that fall within its jurisdiction.

\footnotetext{
Author affiliations

${ }^{1}$ Psychiatry Research, Dartmouth-Hitchcock Medical Center, Lebanon, New Hampshire, USA

${ }^{2}$ Geisel School of Medicine, Dartmouth College, Hanover, New Hampshire, USA

${ }^{3}$ School of Nursing, University of Alabama at Birmingham, Birmingham, Alabama, USA

${ }^{4}$ Division of Preventive Medicine, University of Alabama at Birmingham, Birmingham, Alabama, USA

${ }^{5}$ Department of Hematology Oncology, Dartmouth-Hitchcock Medical Center, Lebanon, New Hampshire, USA

${ }^{6}$ Radiation Oncology, University of Alabama at Birmingham, Birmingham, Alabama, USA

${ }^{7}$ Medicine, Divisions of Hematology and Oncology, and Geriatrics, Gerontology, and Palliative Care, University of Alabama at Birmingham, Birmingham, Alabama, USA

${ }^{8}$ Physical Medicine and Rehabilitation, Dartmouth-Hitchcock Medical Center, Lebanon, New Hampshire, USA

${ }^{9}$ Occupational Therapy, Boston University College of Health and Rehabilitation Sciences Sargent College, Boston, Massachusetts, USA

${ }^{10}$ Occupational Therapy, University of Alabama at Birmingham, Birmingham, Alabama, USA
}

${ }^{11}$ Department of Medicine, Division of Hematology and Oncology, University of Alabama at Birmingham, Birmingham, Alabama, USA

Acknowledgements The research reported in this protocol uses resources of the Dartmouth Clinical and Translational Science Institute, under award number UL1TR001086 from the National Center for Advancing Translational Sciences (NCATS) of the National Institutes of Health (NIH). Preparation of this manuscript was also supported in part by the National Institute of Mental Health (T32 MH073553, PI: Bruce, Fellow: Stevens).The content is solely the responsibility of the authors and does not represent the official views of the National Institutes of Health.

Contributors KDL and MTH conceived the study and KDL, MTH, MB, MAB, AA, $M P, M C$ and $K K$ contributed to the study design. Acquisition of data was performed by KDL, MAB, TG, DE, ANM, JLM, RMN, KET, MEC, SMdA, DZC, JE, SAl, JD and GR. CJS and KDL drafted the manuscript and all authors provided comments and feedback for improving the manuscript. All authors approved the final version of the manuscript and are responsible for their contributions.

Funding Research reported in this publication was supported by the National Cancer Institute of the National Institutes of Health under Award Number R01CA225792. The content is solely the responsibility of the authors and does not necessarily represent the official views of the National Institutes of Health.

Disclaimer The funder did not have a role in the design or conduct of the study and the authors have ultimate authority over study activities. The content is solely the responsibility of the authors and does not necessarily represent the official views of the National Cancer Institute.

Competing interests GR reports grants and other from Genetech, grants and other from Pfizer, grants and other from Carevive, outside the submitted work.

Patient consent for publication Not required.

Ethics approval This protocol is approved by the Committee for the Protection of Human Subjects at Dartmouth College, acting as the single IRB of record for both study sites.

Provenance and peer review Not commissioned; peer reviewed for ethical and funding approval prior to submission.

Open access This is an open access article distributed in accordance with the Creative Commons Attribution Non Commercial (CC BY-NC 4.0) license, which permits others to distribute, remix, adapt, build upon this work non-commercially, and license their derivative works on different terms, provided the original work is properly cited, appropriate credit is given, any changes made indicated, and the use is non-commercial. See: http://creativecommons.org/licenses/by-nc/4.0/.

ORCID iDs

Courtney J Stevens http://orcid.org/0000-0002-5696-4904

Kathleen Doyle Lyons http://orcid.org/0000-0002-8895-4612

\section{REFERENCES}

1 American Cancer Society. Cancer facts and figures 2016 Atlanta, GA, 2016. Available: http://www.cancer.org/research/cancerfactsstati stics/cancerfactsfigures2016/index

2 Arndt V, Merx H, Stegmaier C, et al. Persistence of restrictions in quality of life from the first to the third year after diagnosis in women with breast cancer. JCO 2005;23:4945-53.

3 Ness K, Wall M, Oakes J, et al. Physical performance limitations and participation restrictions among cancer survivors: a populationbased study. Ann Epidemiol 2006;16:197-205.

4 Williamson GM. Extending the activity restriction model of depressed affect: evidence from a sample of breast cancer patients. Health Psychol 2000;19:339-47.

5 Penttinen HM, Saarto T, Kellokumpu-Lehtinen P, et al. Quality of life and physical performance and activity of breast cancer patients after adjuvant treatments. Psychooncology 2011;20:1211-20.

6 Braithwaite D, Satariano WA, Sternfeld B, et al. Long-Term prognostic role of functional limitations among women with breast cancer. J Natl Cancer Inst 2010;102:1468-77.

7 DiSipio T, Hayes S, Battistutta D, et al. Patterns, correlates, and prognostic significance of quality of life following breast cancer. Psychooncology 2011;20:1084-91.

8 Zheng Z, Yabroff KR, Guy GP, et al. Annual medical expenditure and productivity loss among colorectal, female breast, and prostate cancer survivors in the United States. J Natl Cancer Inst 2016;108:djv382. 
9 Fu AZ, Chen L, Sullivan SD, et al. Absenteeism and short-term disability associated with breast cancer. Breast Cancer Res Treat 2011;130:235-42.

10 Steiner JF, Cavender TA, Main DS, et al. Assessing the impact of cancer on work outcomes. Cancer 2004;101:1703-11.

11 Egan MY, McEwen S, Sikora L, et al. Rehabilitation following cancer treatment. Disabil Rehabil 2013;35:2245-58.

12 Loh SY, Musa AN. Methods to improve rehabilitation of patients following breast cancer surgery: a review of systematic reviews. Breast Cancer 2015;7:81-98.

13 Hopko DR, Lejuez CW, Ruggiero KJ, et al. Contemporary behavioral activation treatments for depression: procedures, principles, and progress. Clin Psychol Rev 2003;23:699-717.

14 Cuijpers P, van Straten A, Warmerdam L. Behavioral activation treatments of depression: a meta-analysis. Clin Psychol Rev 2007;27:318-26.

15 Cuijpers P, van Straten A, Warmerdam L. Problem solving therapies for depression: a meta-analysis. Eur Psychiatry 2007;22:9-15.

16 Hegel MT, Arean PA. Problem-Solving treatment for primary care: a treatment manual for depression, project impact. Hanover, $\mathrm{NH}$ : Dartmouth College, 2003.

17 Baum CM, Christiansen CH. Person-environment-occupationperformance: An occupation-based framework for practice. In: Christiansen CH, Baum CM, Bass-Haugen J, eds. Occupational therapy: performance, participation, and well-being. Thorofare, $\mathrm{NJ}$ : SLACK, Inc, 2005: 243-66.

18 Hegel MT, Lyons KD, Hull JG, et al. Feasibility study of a randomized controlled trial of a telephone-delivered problem-solvingoccupational therapy intervention to reduce participation restrictions in rural breast cancer survivors undergoing chemotherapy. Psychooncology 2011;20:1092-101.

19 Lyons KD, Erickson KS, Hegel MT. Problem-Solving strategies of women undergoing chemotherapy for breast cancer. Can J Occup Ther 2012:79:33-40.

20 Lyons KD, Hull JG, Kaufman PA, et al. Development and initial evaluation of a telephone-delivered, behavioral activation, and problem-solving treatment program to address functional goals of breast cancer survivors. J Psychosoc Oncol 2015;33:199-218.

21 Lyons KD, Svensborn IA, Kornblith AB, et al. A content analysis of recovery strategies of breast cancer survivors enrolled in a goalsetting intervention. OTJR: Occupation, Participation and Health 2015;35:73-80.

22 Mundt JC, Marks IM, Shear MK, et al. The work and social adjustment scale: a simple measure of impairment in functioning. $\mathrm{Br}$ J Psychiatry 2002;180:461-4.

23 American Cancer Society. Cancer treatment and survivorship facts and figures 2014-2015. Atlanta: American Cancer Society, 2015.

24 Callahan CM, Unverzagt FW, Hui SL, et al. Six-item screener to identify cognitive impairment among potential subjects for clinical research. Med Care 2002;40:771-81.

25 Carver CS, Scheier MF. On the self-regulation of behavior. anonymous, editor. New York, NY, US: Cambridge University Press, 1998: Xx p.

26 Carver CS, Smith RG, Petronis VM, et al. Quality of life among long-term survivors of breast cancer: different types of antecedents predict different classes of outcomes. Psychooncology 2006;15:749-58

27 Carver CS, Pozo C, Harris SD, et al. How coping mediates the effect of optimism on distress: a study of women with early stage breast cancer. J Pers Soc Psychol 1993;65:375-90.

28 Hull J. Modeling the Structure of Self-Knowledge and the Dynamics of Self-Regulation. In: Tesser A, Stapel DA, Wood JV, eds. Self and motivation: emerging psychological perspectives. Washington, DC: American Psychological Association, 2002: 173-206.

29 Wrosch C, Sabiston CM. Goal adjustment, physical and sedentary activity, and well-being and health among breast cancer survivors. Psychooncology 2013;22:581-9.

30 Law M, Baptiste S, Carswell A, et al. Canadian occupational performance measure. 5th edn. Toronto: Canadian Association of Occupational Therapists, 2014.

31 Popp L, Schneider S. Attention placebo control in randomized controlled trials of psychosocial interventions: theory and practice. Trials 2015;16:150.

32 Harris PA, Taylor R, Thielke R, et al. Research electronic data capture (REDCap)-A metadata-driven methodology and workflow process for providing translational research informatics support. J Biomed Inform 2009:42:377-81.
33 Charlson ME, Pompei P, Ales KL, et al. A new method of classifying prognostic comorbidity in longitudinal studies: development and validation. J Chronic Dis 1987;40:373-83.

34 Martin Ginis KA, Evans MB, Mortenson WB, et al. Broadening the conceptualization of participation of persons with physica disabilities: a configurative review and recommendations. Arch Phys Med Rehabil 2017:98:395-402.

35 Castel LD, Williams KA, Bosworth HB, et al. Content validity in the PROMIS social-health domain: a qualitative analysis of focus-group data. Qual Life Res 2008;17:737-49.

36 Hahn EA, DeVellis RF, Bode RK, et al. Measuring social health in the patient-reported outcomes measurement information system (PROMIS): item bank development and testing. Qual Life Res 2010;19:1035-44.

37 Jensen RE, Moinpour CM, Potosky AL, et al. Responsiveness of 8 patient-reported outcomes measurement information system (PROMIS) measures in a large, community-based cancer study cohort. Cancer 2017;123:327-35.

38 Ekwueme DU, Yabroff KR, Guy GP, et al. Medical costs and productivity losses of cancer survivors--United States, 2008-2011. MMWR Morb Mortal Wkly Rep 2014:63:505-10.

39 Lerner D, Reed JI, Massarotti E, et al. The work limitations questionnaire's validity and reliability among patients with osteoarthritis. J Clin Epidemiol 2002;55:197-208.

40 Tamminga SJ, Verbeek JHAM, Frings-Dresen MHW, et al. Measurement properties of the work limitations questionnaire were sufficient among cancer survivors. Qual Life Res 2014;23:515-25.

41 Leensen MCJ, Groeneveld IF, Heide Ivander, et al. Return to work of cancer patients after a multidisciplinary intervention including occupational counselling and physical exercise in cancer patients: a prospective study in the Netherlands. BMJ Open 2017;7:e014746.

42 Feuerstein M, Hansen JA, Calvio LC, et al. Work productivity in brain tumor survivors. J Occup Environ Med 2007;49:803-11.

43 Lerner D, Amick BC, Lee JC, et al. Relationship of employeereported work limitations to work productivity. Med Care 2003;41:649-59.

44 Brady MJ, Cella DF, Mo F, et al. Reliability and validity of the functional assessment of cancer Therapy-Breast quality-of-life instrument. JCO 1997;15:974-86.

45 Coster S, Poole K, Fallowfield LJ. The validation of a quality of life scale to assess the impact of arm morbidity in breast cancer patients post-operatively. Breast Cancer Res Treat 2001;68:273-82.

46 Carver CS. You want to measure coping but your protocol' too long: Consider the brief cope. Int J Behav Med 1997;4:92-100.

47 Fillion L, Kovacs AH, Gagnon P, et al. Validation of the shortened cope for use with breast cancer patients undergoing radiation therapy. Current Psychology 2002;21:17-34.

48 Wrosch C, Scheier MF, Miller GE, et al. Adaptive self-regulation of unattainable goals: goal disengagement, goal reengagement, and subjective well-being. Personality and Social Psychology Bulletin 2003;29:1494-508.

49 Zigmond AS, Snaith RP. The hospital anxiety and depression scale. Acta Psychiatr Scand 1983;67:361-70.

50 Szanton SL, Xue Q-L, Leff B, et al. Effect of a Biobehavioral environmental approach on disability among low-income older adults: a randomized clinical TrialEffect of a Biobehavioral environmental approach on disability among older AdultsEffect of a Biobehavioral environmental approach on disability among older adults. JAMA Internal Medicine 2019;179:204-11.

51 Brown H, Prescott R. Applied mixed models in medicine. 3rd Ed. Chichester, England: Wiley, 2015.

52 van Buuren S. Flexible imputation of missing data. Boca Raton, FL: CRC Press, 2012.

53 Buuren Svan, Groothuis-Oudshoorn K. mice : Multivariate Imputation by Chained Equations in R. J Stat Softw 2011;45:67.

54 Molenberghs G, Kenward M. Missing data in clinical studies. Hoboken: Wiley, 2007.

55 Benjamini Y, Hochberg Y. Controlling the false discovery rate: a practical and powerful approach to multiple testing. Journal of the Royal Statistical Society: Series B 1995;57:289-300.

56 Strobl C, Malley J, Tutz G. An introduction to recursive partitioning: rationale, application, and characteristics of classification and regression trees, bagging, and random forests. Psychol Methods 2009;14:323-48

57 Hothorn T, Hornik K, Zeileis A. Unbiased recursive partitioning: a conditional inference framework. Journal of Computational and Graphical Statistics 2006;15:651-74. 